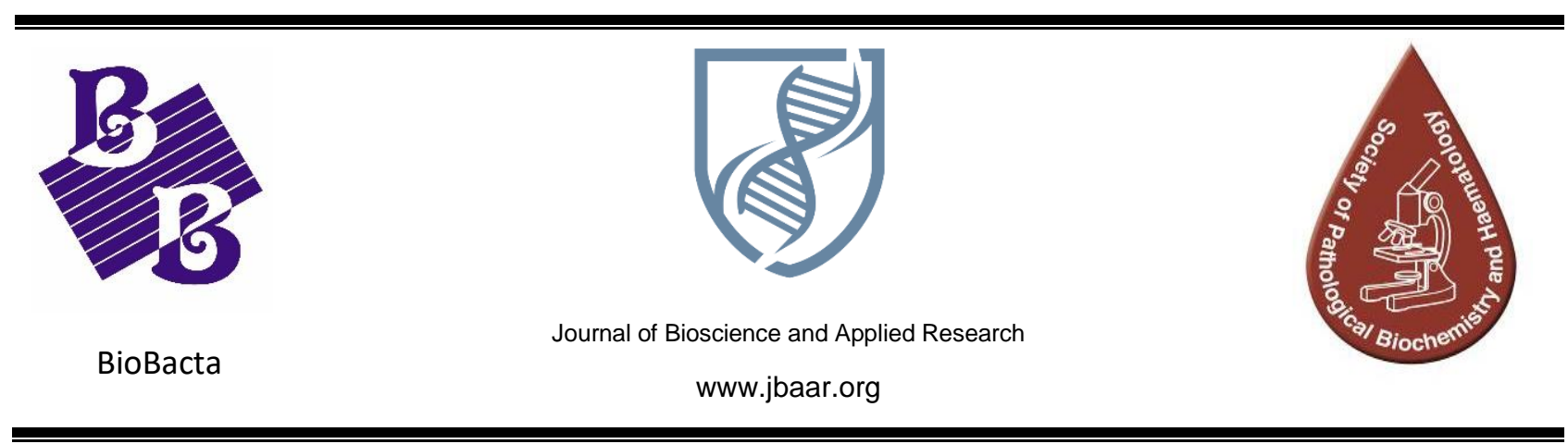

\title{
Diversity of Mix Microalgae in Fish Tanks under Different Weather Conditions
}

\author{
Hayfaa Mohammed Sahab, Hishamuddin Omer, Ahmad Ismail, Noor Amal Mohamed \\ Department of Biology, Faculty of Science, University Putra Malaysia \\ 43400, UPM Serdang, Selangor Darul Ehsan, Malaysia \\ Correspondent author: shh20072000@yahoo.com
}

DOI: 10.21608/jbaar.2018.155803

\begin{abstract}
One of the biggest problems facing the world today is global warming. Many scientists believe that our production of carbon dioxide and other greenhouse gases contributed to the warming of the atmosphere and this effecting most living organisms on earth including microalgae. Therefore, this study aims to observe the changes in the diversity of mixed microalgae communities in different stocking densities of fish under different weather conditions within effects of environmental parameters on microalgae. Phytoplankton samples were +collected from five tanks, one tank is a control tank without fish and four tanks with different densities of fish (10, 20, 40, and 80 fishes) respectively under different weather conditions for microalgal diversity study. Weather conditions and rainfall were recorded daily. Microalgae samples were collected from fish were identified, enumerated and the diversity index determined based on Shannon, Richness, Evenness, and Simpson. There are 56 microalgal taxa found in the outdoor fish tanks belonging to 6 divisions represented by Chlorophyta (38) followed by Bacillariophyta (6), Cyanophyta (6), Dinophyta (3), Euglenophyta (2), and Crysophyta (1) in three weather conditions. The highest number of taxa recorded was 41 in tank 2 (20 fish), 39 taxa were observed in tank 3 (40 fish) followed by 34 taxa in tank 4 ( 80 fish), 33 taxa in tank 1 (10 fish), and 13 taxa in control tank without fish. In wet weather, the number of taxa was 25 followed by mixed weather 24 and dry weather with 22 taxa. Favorable water quality during wet weather is probably led to the highest species occurrence. Control tank without fish has 13 species represented by four microalgal divisions. Chlorophyta was the most common division in all tanks with 33 taxa in tank 1 (10 fishes), 41 taxa in tank 2 (20 fish), 39 taxa in tank 3 (40 fish), and 33 taxa in tank 4 (80 fish) in three weather conditions. The highest number of species occurred in wet weather and the lowest species occurrence in dry weather conditions. The most common and dominant species was Pandorina morum found in all tanks in different weather conditions. This study concluded that the fish stocking density and weather conditions influenced microalgal species composition and diversity.
\end{abstract}

Keywords: Fish stocking density, weather conditions, Microalgae species, and Diversity 


\section{Introduction}

Presently the most serious and important problem humankind has ever had to face might be global warming with disastrous consequences and costly adverse effects (Shahzad, 2015). Its impact is wide-ranging such as rising sea levels rise, extreme weather effects, loss of habitat, and many more. Global warming and climate change occurred by natural and human causes. Most of the causes were due to the release of $\mathrm{CO}_{2}$ to the atmosphere. The best way to reduce global warming is cutting down anthropogenic emission of greenhouse gases. Numerous past and present studies have indicated that microalgae can potentially reduce greenhouse gas from coal-fired power plant and other carbon intensive industrial activities. This is possible because a microalga is small undifferentiated body with fast growth, able to live in diverse habitat, high accumulation of lipid and carbohydrate during starvation conditions and higher productivity (Bahadar et al., 2013). Microalgae can grow 20 or 30 times faster than traditional food crops, and has no need to compete for arable land (Sivasubramanian, 2016). The study on diversity of microalgae is very important to know what type of algae can be used to improve water quality. Most of the phytoplanktons can exist in open surface waters of lakes, rivers and oceans. Microalgae are also commonly found in pools, water storage tanks, and fish tanks. Many reports revealed that the microalgae abundance in ponds vary with changes in environmental factors such as light, temperature, pH and salinity (Shaari et al., 2011). Unfortunately most of the studies on microalgae are conducted in the laboratory (Ruangsomboon, 2012). Another advantage is that microalgae are capable of both carbon assimilation and $\mathrm{O}_{2}$ productivity, thereby enhancing productivity in variety of environment (Makandar \& Bhatnagar, 2010). The oldest documented use of microalgae was 2000 years ago, when the Chinese used cyanobacteria Nostock a food source during famine (Priyadarshani \& Rath 2012). Today cultured microalgae is used as food supplement for humans, feed for land-based farm animal, and as feed for cultured aquatic species such as molluscs and the early larval stages of fish and crustaceans (Barnabé, 1994). Algae can also produce a variety of high value compounds such as fine chemicals, biofertilizer and biodiesel, without contributing to atmospheric carbon dioxide (AbdelRaouf et al., 2012). Under favourable conditions that include adequate light availability, warm waters, and high nutrients levels, algae can rapidly grow multiply and if unchecked may cause blooms. Algal bloom can harm the aquatic environments by blocking sunlight and depleting $\mathrm{O} 2$ required by other aquatic organisms, restricting their growth and survival (EPA, 2013).

This diversity provides man with economic and aesthetic benefits. Climate change is predicted to change many environmental conditions that could affect the natural properties of freshwater. Biodiversity is the controlling key factor in ecology, directly linked with the functioning and regulation of the ecosystems. Measurement of environmental changes and toxicants can be achieved through using microalgae as a biological tool (Cardozo et al., 2007). Therefore, any changes in microalgae species can occur under different conditions as a response to various environmental factors. The production of algae from fish tanks considered as one of the axes of economic development because the algae are a rich source of amino and fatty acids and are easily absorbed by fish. This is necessary to complete and continue the healthy food chain. Therefore, this study aims to observe the changes in the diversity of mixed microalgae communities in different stocking densities of fish under different weather conditions within effects of environmental parameters on microalgae.

\section{Materials and Methods}

The experiment was conducted at the Tilapia pond the fish hatchery unit of University Agriculture Park (UAP) at the University of Putra Malaysia. Five fiberglass tanks rectangle tanks each with dimensions $($ Length $=220 \mathrm{~cm}$, Width $=98 \mathrm{~cm}$, and Depth $=40 \mathrm{~cm})$, filled with 1000 litter dechlorinated tap water and different stocking density of red Nile tilapia (Oreochromis niloticus), tank 1(10 fish), tank 2 (20 fish), tank 3 (40 fish), tank 4 (80 fish) and the fifth tank is controlled without fish. The fingerlings were approximately $1.074 \mathrm{~g}$ in weight and $3 \mathrm{~cm}$ in length. The commercial starter floating fish feed was calculated according to (New, 1987). Water samples were collected every two days from November 2014 to 
February 2015. Temperature, dissolved oxygen, $\mathrm{PH}$, salinity, conductivity, and total dissolved solids were determined by Yellow Spring Instrument by multi-parameter probe model (YSI-556 MPS) (Harun et al., 2015). Water transparency was measured by Secchi disk ( $20 \mathrm{~cm}$ diameter). The air temperature and light intensity was measured using the light meter Licor model (L1-250). The temperature, light intensity, and weather state were observed and recorded daily, three times a day at 8.00-9.00am, 12.00-1.00 am 4,00-5.00pm. The rainfall was determined using a rain gauge after a time of rain by using a 5L size bottle with a fixed funnel (Goldstein, 2002). The calculation of rainfall based on a formula (Raghunath, 2006):

Rainfall gauging $(\mathrm{mm})=($ Collected water volume $) /$ (funnel surface area/bottle base area)

Samples of microalgae were collected every two days in different weather conditions (wet, mix, dry) by using five plastic bottles for five tanks, triplicate sampling of $500 \mathrm{ml}$ water samples. All samples were preserved by Glutaraldehyde solution (Kumar, 2012), and then the samples were left constant for 48 hours to allow microalgae to settle down (Edler and Elbrächter, 2010). Then, the $400 \mathrm{ml}$ of the upper water of the bottle were removed, and then the remaining $100 \mathrm{ml}$ were used for identification and enumeration using a compound microscope at a magnification of 400x. One drop of the sample was placed into a haemocytometer counting chamber.
Each sample was counted three times and then takes average. All species of algae were identified according to a key of freshwater algae (Bellinger and Sigee, 2010) and microalgae were counted by Olympus light microscope at a magnification of 400x. The diversity of microalgae was done by the Shannon-Weaver index (Shannon and Weiner, 1949), Simpson index (Simpson, 1949), Evenness, and species richness (Margalef, 1958).

\section{Statistical analysis}

The data analyzed by using Microsoft Excel and statistical analysis (version 20) one-way ANOVA to indicate the significance of variance of physical environmental parameters, and for water quality parameters and species diversity using factorial ANOVA to determine the difference between treatments.

\section{Results}

Malaysian weather is very complex to define into a distinct season. Cloudy and sunny sky and rains can occur at any time of the day. Weather conditions were classified depending on the daily weather recording of the study period. The weather conditions can be classified into three weather conditions wet, mix, and dry based on scores represented in table 1 and there were 9 cycles throughout the study comprising of three weather conditions each. The weather conditions scoring classified as: 1-4 (wet), 5-7 (mix), 7-10 (dry).

Table 1: The weekly weather scoring during the study period (Weather score from 1 to 10,1-4. denote Wet weather, $5-7$ denote mixed weather and 7-10 denote Dry weather)

\begin{tabular}{cccccccccc}
\hline \multirow{2}{*}{ Days } & \multicolumn{10}{c}{ Culture Cycles during the study period } \\
& C1 & C2 & C3 & C4 & C5 & C6 & C7 & C8 & C9 \\
\hline 1 & 2 & 6 & 6 & 3 & 3 & 6 & 8 & 8 & 2 \\
2 & 5 & 7 & 4 & 6 & 4 & 1 & 7 & 8 & 5 \\
3 & 7 & 5 & 1 & 5 & 4 & 1 & 10 & 9 & 10 \\
4 & 5 & 1 & 3 & 5 & 4 & 2 & 6 & 9 & 7 \\
5 & 7 & 4 & 9 & 6 & 4 & 6 & 7 & 8 & 6 \\
6 & 1 & 7 & 5 & 2 & 4 & 4 & 4 & 7 & 8 \\
7 & 3 & 6 & 2 & 6 & 7 & 5 & 2 & 7 & 6 \\
8 & 4 & 6 & 2 & 3 & 3 & 6 & 7 & 8 & 9 \\
9 & 7 & 5 & 1 & 5 & 3 & 1 & 10 & 10 & 8 \\
10 & 10 & 4 & 2 & 1 & 2 & 7 & 9 & 8 & 9 \\
\hline Mean & 5.1 & 5.6 & 3.5 & 4.2 & 6.1 & 3.9 & 7 & 8.2 & 7 \\
\hline Category & Mix & Mix & Wet & Wet & Mix & Wet & Dry & Dry & Dry \\
\hline
\end{tabular}


Based on table (2) there is a significant difference $(\mathrm{P}<0.05)$ between weather conditions in temperature and light intensity. The rainfall was a significant difference between weathers. As it is shown throughout table 2 there was a significant difference between weather conditions, the wet weather recorded a higher rate $605.31 \mathrm{ml}, 438.72$ $\mathrm{ml}, 431.58 \mathrm{ml}$ in three cycles followed by mixed weather $232.77 \mathrm{ml}, 157.28 \mathrm{ml}, 85.76 \mathrm{ml}$, while the rate of rainfall was lower in dry weather $0,40.8 \mathrm{ml}$, and $53.2 \mathrm{ml}$.

Table 2: Average of air temperature, light intensity and rainfall (* Temperature category: 26.79-29.87, **Light intensity category: 217.05-688.90,***Rain category: 53.20-605.31)

\begin{tabular}{|c|c|c|c|c|c|c|c|c|c|}
\hline $\begin{array}{l}\text { Cycles } \\
\text { Category }\end{array}$ & $\begin{array}{l}\text { C1 } \\
\text { Mix }\end{array}$ & $\begin{array}{l}\text { C2 } \\
\text { Mix }\end{array}$ & $\begin{array}{l}\text { C3 } \\
\text { Wet }\end{array}$ & $\begin{array}{l}\text { C4 } \\
\text { Wet }\end{array}$ & $\begin{array}{l}\text { C5 } \\
\text { Mix }\end{array}$ & $\begin{array}{l}\text { C6 } \\
\text { Wet }\end{array}$ & $\begin{array}{l}\text { C7 } \\
\text { Dry }\end{array}$ & $\begin{array}{l}\text { C8 } \\
\text { Dry }\end{array}$ & $\begin{array}{l}\text { C9 } \\
\text { Dry }\end{array}$ \\
\hline $\begin{array}{c}\text { Temperature } \\
\left(\mathrm{C}^{\circ}\right)^{*} \\
\text { Light }\end{array}$ & $\begin{array}{l}29.10 \\
\pm 0.42\end{array}$ & $\begin{array}{l}28.20 \\
\pm 0.35\end{array}$ & $\begin{array}{l}28.60 \\
\pm 0.40\end{array}$ & $\begin{array}{l}26.79 \\
\pm 0.79\end{array}$ & $\begin{array}{l}28.96 \\
\pm 0.37\end{array}$ & $\begin{array}{l}27.09 \\
\pm 0.40\end{array}$ & $\begin{array}{l}28.08 \\
\pm 0.54\end{array}$ & $\begin{array}{l}29.87 \\
\pm 0.54\end{array}$ & $\begin{array}{l}29.40 \\
\pm 0.46\end{array}$ \\
\hline $\begin{array}{c}\text { intensity }(\mu \mathrm{mol} \mathrm{m} \\
\left.{ }^{2} \mathrm{~s}^{-1}\right) \\
* *\end{array}$ & $\begin{array}{r}505.80 \\
\pm 68.93\end{array}$ & $\begin{array}{l}540.26 \\
\pm 60.02\end{array}$ & $\begin{array}{l}466.85 \\
\pm 53.45\end{array}$ & $\begin{array}{l}217.05 \\
\pm 21.09\end{array}$ & $\begin{array}{l}522.47 \\
\pm 58.66\end{array}$ & $\begin{array}{l}287.28 \\
\pm 43.96\end{array}$ & $\begin{array}{l}562.34 \\
\pm 76.44\end{array}$ & $\begin{array}{r}688.90 \\
\pm 63.73\end{array}$ & $\begin{array}{l}588.91 \\
\pm 75.67\end{array}$ \\
\hline Rainfall $(\mathrm{ml})^{\star * *}$ & $\begin{array}{l}232.77 \\
\pm 89.37\end{array}$ & $\begin{array}{c}85.76 \\
\pm 52.54\end{array}$ & $\begin{array}{c}605.31 \\
\pm 276.27\end{array}$ & $\begin{array}{r}431.58 \\
\pm 158.76\end{array}$ & $\begin{array}{l}157.28 \\
\pm 55.95\end{array}$ & $\begin{array}{c}438.72 \\
\pm 150.16\end{array}$ & $\begin{array}{c}40.80 \\
\pm 24.80\end{array}$ & $\begin{array}{c}0.00 \\
\pm 0.00\end{array}$ & $\begin{array}{c}53.20 \\
\pm 16.02\end{array}$ \\
\hline Category & Mix & Mix & Wet & Wet & Mix & Wet & Dry & Dry & Dry \\
\hline
\end{tabular}

Table 3 indicated different aspect of weather condition that exerts influence on water quality physical parameters with a different stocking density of fish in three cultivation cycles. Water temperature was significantly different $(\mathrm{p}<0.05)$ in different weather conditions but there was no significant difference $(\mathrm{p}>0.05)$ between the cycles. The highest temperature was $29.24 \pm 0.38$, $27.48 \pm 0.07^{\circ} \mathrm{C}$ during dry and mixed weather respectively in tank 4 (80 fish), whilst the lowest temperature was $26.87 \pm \pm^{\circ} \mathrm{C}$ recorded in wet weather in control (without fish) with a range of 26.87 to $29.24{ }^{\circ} \mathrm{C}$.

$\mathrm{pH}$ is a measure of hydrogen ions or acidity in the water which is very important for biotic and abiotic features of aquatic systems. $\mathrm{pH}$ value often fluctuated within the cultivation periods. The $\mathrm{pH}$ values fluctuate marginally at $7.35 \pm 0.01$, $7.25 \pm 0.02$, and $7.18 \pm 0.05$ during mix, wet, and dry weather conditions respectively. The $\mathrm{pH}$ of the water was significantly different $(\mathrm{p}<0.05)$ between control and other tanks but there were no significant differences $(p>0.05)$ between culture cycles and tanks with fish.

Electrical conductivity (EC) is a measure of water's capability to pass electrical flow. This ability is directly related to the concentration of ions in the water. There were no significant differences ( $p>0.05)$ of EC between culture cycles and tanks (with fish and control) but there were significantly different $(\mathrm{p}<0.05)$ within cultivation periods and weather conditions ranging between 0.13 to 0.17 $\mathrm{mS} / \mathrm{cm}$ and the average of $0.16 \pm 0.00,0.13 \pm 0.00$ and $0.17 \pm 0.00$ in the mix, wet and dry weather conditions respectively.

Total dissolved solids (TDS) are the term used to describe the presence of inorganic salts and small amounts of organic matter present in water. Significant seasonal variations in total dissolved solids $(\mathrm{p}<0.05)$ were observed. The highest value was $0.10 \mathrm{mg} / \mathrm{L}$ in the mix and dry weather and the lowest value was $0.08 \mathrm{mg} / \mathrm{L}$ in wet weather its ranges from 0.08 to $0.10 \mathrm{mg} / \mathrm{L}$. There were no significant differences $(\mathrm{p}>0.05)$ between cultivation cycles, days, and tanks.

The mean value of dissolved oxygen recorded significant variation $(\mathrm{p}<0.05)$ between weather conditions, tanks, and days of cultivation but there was no significant difference $(p>0.05)$ between culture cycles. The highest DO value was $6.69 \mathrm{mg} / \mathrm{L}$ in control in wet weather, while the lowest value was $2.99 \mathrm{mg} / \mathrm{L}$ in tank 4 (80 fish) in dry weather conditions ranged from 2.99 to 6.69 $\mathrm{mg} / \mathrm{L}$. Salinity remained relatively high in the mix 
and dry weather of 0.07 ppt but marginally lower in wet weather $0.06 \mathrm{ppt}$ with no significant difference $(\mathrm{p}>0.05)$ between tanks and cycles but there were significant differences $(p<0.05)$ within the cultivation period.

Secchi disk depth is a measure of the relative clarity of a liquid. Water transparency recorded significant differences $(\mathrm{p}<0.05)$ within weather conditions, tanks and cultivation period $(\mathrm{p}<0.05)$ but there were no significant differences $(p>0.05)$ between cycles. The highest average Secchi disk depth was in control (no fish) $0.40 \mathrm{~m}$ in all weather conditions and the lowest was in tank 4(80 fish) in dry weather $0.12 \mathrm{~m}$ with a range of 0.12 to $0.40 \mathrm{~m}$.

Table 3: The difference between tanks and weather conditions for physical parameters (Average of 3 cycles for every weather)

\begin{tabular}{|c|c|c|c|c|}
\hline \multirow{2}{*}{ Parameters } & \multirow{2}{*}{ Tanks } & \multicolumn{3}{|c|}{ Weather } \\
\hline & & $\operatorname{Mix}{ }^{b}$ & Wet ${ }^{a}$ & Dry ${ }^{c}$ \\
\hline \multirow{6}{*}{$\begin{array}{c}\text { Temperature } \\
\left({ }^{\circ} \mathrm{C}\right)\end{array}$} & Control & $27.31 \pm 0.03^{a}$ & $26.87 \pm 0.11^{a}$ & $28.95 \pm 0.19^{a}$ \\
\hline & T1 (10 Fish) & $27.42 \pm 0.02^{a}$ & $26.98 \pm 0.04^{a}$ & $29.09 \pm 0.12^{a}$ \\
\hline & T2 (20 Fish) & $27.42 \pm 0.03^{a}$ & $26.89 \pm 0.02^{a}$ & $29.00 \pm 0.05^{a}$ \\
\hline & T3 (40 Fish) & $27.31 \pm 0.04^{\mathrm{a}}$ & $27.00 \pm 0.04^{a}$ & $29.05 \pm 0.22^{a}$ \\
\hline & T4 (80 Fish) & $27.48 \pm 0.07^{a}$ & $26.98 \pm 0.05^{a}$ & $29.24 \pm 0.38^{a}$ \\
\hline & & Mix ${ }^{b}$ & Wet $^{a}$ & Dry $^{b}$ \\
\hline \multirow{6}{*}{$\mathrm{pH}$} & Control & $7.33 \pm 0.01^{b}$ & $7.25 \pm 0.02^{b}$ & $7.18 \pm 0.05^{b}$ \\
\hline & T1 (10 Fish) & $6.94 \pm 0.02^{\mathrm{a}}$ & $6.76 \pm 0.03^{a}$ & $7.41 \pm 0.04^{\mathrm{a}}$ \\
\hline & T2 (20 Fish) & $7.35 \pm 0.04^{a}$ & $6.76 \pm 0.07^{a}$ & $7.03 \pm 0.04^{a}$ \\
\hline & T3 (40 Fish) & $6.97 \pm 0.02^{a}$ & $6.93 \pm 0.10^{a}$ & $6.97 \pm 0.02^{a}$ \\
\hline & T4 (80 Fish) & $6.95 \pm 0.03^{a}$ & $7.13 \pm 0.11^{a}$ & $7.03 \pm 0.04^{\mathrm{a}}$ \\
\hline & & $\operatorname{Mix}{ }^{b}$ & Wet ${ }^{a}$ & Dry $^{c}$ \\
\hline \multirow{6}{*}{$\begin{array}{l}\text { Conductivity } \\
\left(\mathrm{mScm}^{-1}\right)\end{array}$} & Control & $0.15 \pm 0.00^{a}$ & $0.13 \pm 0.00^{a}$ & $0.17 \pm 0.00^{a}$ \\
\hline & T1 (10 Fish) & $0.16 \pm 0.00^{a}$ & $0.13 \pm 0.00^{a}$ & $0.17 \pm 0.00^{a}$ \\
\hline & T2 (20 Fish) & $0.16 \pm 0.00^{a}$ & $0.13 \pm 0.00^{a}$ & $0.17 \pm 0.00^{\mathrm{a}}$ \\
\hline & T3 (40 Fish) & $0.16 \pm 0.00^{\mathrm{a}}$ & $0.14 \pm 0.00^{a}$ & $0.17 \pm 0.00^{\mathrm{a}}$ \\
\hline & T4 (80 Fish) & $0.16 \pm 0.00^{\mathrm{a}}$ & $0.14 \pm 0.00^{a}$ & $0.17 \pm 0.00^{\mathrm{a}}$ \\
\hline & & Mix ${ }^{b}$ & Wet $^{a}$ & Dry $^{c}$ \\
\hline \multirow{6}{*}{$\begin{array}{l}\text { Total } \\
\text { dissolved } \\
\text { soilds } \\
\text { (mg L-1) }\end{array}$} & Control & $0.10 \pm 0.00^{a b}$ & $0.08 \pm 0.00^{a b}$ & $0.10 \pm 0.00^{a b}$ \\
\hline & T1 (10 Fish) & $0.09 \pm 0.00^{a}$ & $0.08 \pm 0.00^{a}$ & $0.10 \pm 0.00^{a}$ \\
\hline & T2 (20 Fish) & $0.10 \pm 0.00^{a b}$ & $0.08 \pm 0.00^{a b}$ & $0.10 \pm 0.00^{a b}$ \\
\hline & T3 (40 Fish) & $0.09 \pm 0.00^{a b}$ & $0.08 \pm 0.00^{a b}$ & $0.10 \pm 0.00^{a b}$ \\
\hline & T4 (80 Fish) & $0.10 \pm 0.00^{b}$ & $0.09 \pm 0.00^{b}$ & $0.10 \pm 0.00^{b}$ \\
\hline & & Mix ${ }^{b}$ & Wet $^{\mathrm{c}}$ & Dry $^{a}$ \\
\hline \multirow{6}{*}{$\begin{array}{l}\text { Dissolved } \\
\text { Oxygen } \\
\text { (mg L-1) }\end{array}$} & Control & $5.16 \pm 0.04^{c}$ & $6.69 \pm 0.08^{c}$ & $4.46 \pm 0.10^{c}$ \\
\hline & T1 (10 Fish) & $5.15 \pm 0.08^{c}$ & $6.42 \pm 0.19^{c}$ & $4.35 \pm 0.15^{c}$ \\
\hline & T2 (20 Fish) & $4.95 \pm 0.14^{b}$ & $6.35 \pm 0.23^{b}$ & $4.00 \pm 0.03^{b}$ \\
\hline & T3 (40 Fish) & $4.48 \pm 0.16^{b}$ & $5.74 \pm 0.03^{b}$ & $3.64 \pm 0.09^{b}$ \\
\hline & T4 (80 Fish) & $4.10 \pm 0.22^{\mathrm{a}}$ & $5.30 \pm 0.03^{a}$ & $2.99 \pm 0.03^{a}$ \\
\hline & & Mix ${ }^{b}$ & Wet $^{a}$ & Dry ${ }^{b}$ \\
\hline \multirow{6}{*}{$\begin{array}{l}\text { Salinity } \\
\text { (ppt) }\end{array}$} & Control & $0.07 \pm 0.00^{a}$ & $0.06 \pm 0.00^{a}$ & $0.06 \pm 0.00^{a}$ \\
\hline & T1 (10 Fish) & $0.06 \pm 0.00^{a}$ & $0.06 \pm 0.00^{a}$ & $0.07 \pm 0.00^{a}$ \\
\hline & T2 (20 Fish) & $0.07 \pm 0.00^{a}$ & $0.06 \pm 0.00^{a}$ & $0.06 \pm 0.00^{a}$ \\
\hline & T3 (40 Fish) & $0.07 \pm 0.00^{a}$ & $0.06 \pm 0.00^{a}$ & $0.07 \pm 0.00^{b}$ \\
\hline & T4 (80 Fish) & $0.07 \pm 0.00^{a}$ & $0.06 \pm 0.00^{a}$ & $0.07 \pm 0.00^{b}$ \\
\hline & & Mix ${ }^{b}$ & Wet ${ }^{a}$ & Dry ${ }^{C}$ \\
\hline \multirow{5}{*}{$\begin{array}{l}\text { Secchi disk } \\
\text { depth } \\
\text { (m) }\end{array}$} & Control & $0.40 \pm 0.00^{d}$ & $0.40 \pm 0.00^{d}$ & $0.40 \pm 0.00^{d}$ \\
\hline & T1 (10 Fish) & $0.21 \pm 0.01^{c}$ & $0.29 \pm 0.01^{c}$ & $0.17 \pm 0.01^{c}$ \\
\hline & T2 (20 Fish) & $0.18 \pm 0.01^{b}$ & $0.27 \pm 0.01^{b}$ & $0.14 \pm 0.01^{b}$ \\
\hline & T3 (40 Fish) & $0.17 \pm 0.00^{b}$ & $0.25 \pm 0.01^{b}$ & $0.14 \pm 0.00^{b}$ \\
\hline & T4 (80 Fish) & $0.16 \pm 0.01^{\mathrm{a}}$ & $0.22 \pm 0.00^{a}$ & $0.12 \pm 0.00^{\mathrm{a}}$ \\
\hline
\end{tabular}


In the control tank (Table 4), the total species were 13 under four divisions and division Chlorophyta was the most common. The species of microalgae distributed at 6 species in wet weather, 7 species in mixed weather, and 8 species in dry weather. Most of the abundance in three weather conditions was Navicula sp.

Table 4: List of microalgae species in control tank (without fish) under three weather conditions (Abundance $\mathrm{R}=$ rare occurrence, $<1000$ cells $/ \mathrm{ml}: \mathrm{F}=$ frequent occurrence, $1000-9999$ cells $/ \mathrm{ml} \mathrm{S}=$ subdominant, 10000-99999 cells/ml: $\mathrm{D}=$ dominant, $>100000$ cells $/ \mathrm{ml}$ )

\begin{tabular}{|c|c|c|c|c|}
\hline \multirow{2}{*}{ Division } & \multirow{2}{*}{ Species } & \multicolumn{3}{|c|}{ Weather conditions } \\
\hline & & Wet & Mix & Dry \\
\hline \multirow{8}{*}{ Chlorophyta } & Coelastrum microporum & $\mathrm{S}$ & - & - \\
\hline & Chlorella vulgaris & $\mathrm{F}$ & - & $F$ \\
\hline & $\begin{array}{l}\text { Dictyosphaerium } \\
\text { pulchellum }\end{array}$ & S & - & S \\
\hline & Haematococcus pluvialis & - & $S$ & - \\
\hline & Odeogonium sp. & - & $\mathrm{F}$ & - \\
\hline & Oocystis borgei & $\mathrm{F}$ & - & $F$ \\
\hline & Pandorina morum & - & $\mathrm{F}$ & S \\
\hline & Ulthrix equalise & - & D & $\mathrm{D}$ \\
\hline Dinophyta & Peridenium labeled & - & - & $\mathrm{S}$ \\
\hline \multirow{3}{*}{ Cyanophyta } & Anabena sp. & - & S & S \\
\hline & Microcystis aeruginosa & - & $\mathrm{F}$ & - \\
\hline & Nostoc sp. & S & - & - \\
\hline Bacillariophyta & Navicula sp. & S & $\mathrm{F}$ & $\mathrm{F}$ \\
\hline Total Species & 13 & 6 & 7 & 8 \\
\hline
\end{tabular}


In-tank 1 (10 fish), a total number of 33 algal taxa were identified in three weather conditions which were distributed over 20 species in wet weather, 23 species in mixed weather, and 17 species in dry weather. The 8 species were abundant in three weather conditions which include Chlorella vulgaris, Coelastrum microporum, Dictyosphaerium pulchellum, Golenkinia radiate, Oocystis borgei, Pandorina morum, Scenedesmus quadricauda, and Cocconeis sp. (Table 5).

Table 5: List of microalgae species in tank one (10 fish) in three weather conditions (Abundance $\mathrm{R}=$ rare occurrence, $<1000$ cells/ml: $\mathrm{F}=$ frequent occurrence, $1000-9999$ cells $/ \mathrm{ml} \mathrm{S}=$ subdominant, 10000-99999 cells/ml: $\mathrm{D}=$ dominant, $>100000$ cells/ml)

\begin{tabular}{|c|c|c|c|c|}
\hline \multirow{2}{*}{ Division } & \multirow{2}{*}{ Species } & \multicolumn{3}{|c|}{ Weather conditions } \\
\hline & & Wet & Mix & Dry \\
\hline \multirow{26}{*}{ Chlorophyta } & Ankyra ankora & - & $\mathrm{F}$ & $\mathrm{F}$ \\
\hline & Botryococcus sp. & - & - & $\mathrm{S}$ \\
\hline & Clamydomonas sp. & - & $\mathrm{F}$ & $\mathrm{F}$ \\
\hline & Chlorella vulgaris & S & $\mathrm{F}$ & S \\
\hline & $\begin{array}{l}\text { Chlorogonium } \\
\text { elongatum }\end{array}$ & $S$ & - & - \\
\hline & Coelastrum microporum & S & S & S \\
\hline & $\begin{array}{l}\text { Dictyosphaerium } \\
\text { pulchellum }\end{array}$ & $S$ & $S$ & $S$ \\
\hline & Golenkinia radiate & S & $\mathrm{F}$ & S \\
\hline & Haematococcus pluvialis & - & - & S \\
\hline & Lagerheimia ciliate & S & $\mathrm{F}$ & - \\
\hline & Micractinium pusillum & S & - & - \\
\hline & Monoraphidium & $S$ & S & - \\
\hline & consortium & & & \\
\hline & Odeogonium sp. & S & S & - \\
\hline & Oocystis borgei & S & D & S \\
\hline & Pandorina morum & D & D & $\mathrm{D}$ \\
\hline & Pediastrum duplex & - & $\mathrm{F}$ & $\mathrm{F}$ \\
\hline & Scenedesmus & - & $S$ & - \\
\hline & acuminatus & & & \\
\hline & Scenedesmus acutus & S & - & $\mathrm{F}$ \\
\hline & Scenedesmus obliqus & - & $S$ & - \\
\hline & Scenedesmus obtusus & - & $S$ & S \\
\hline & $\begin{array}{l}\text { Scenedesmus } \\
\text { quadricauda }\end{array}$ & $\mathrm{F}$ & S & S \\
\hline & Staurodesmus dejetus & - & $\mathrm{F}$ & $\mathrm{S}$ \\
\hline & Tetraedron minimum & $\mathrm{F}$ & $\mathrm{F}$ & - \\
\hline & Tetraedron muticum & S & - & - \\
\hline Dinophyta & $\begin{array}{l}\text { Sphaerodinium } \\
\text { woloszynska }\end{array}$ & $\mathrm{R}$ & - & - \\
\hline \multirow{3}{*}{ Bacillariophyta } & Cyclotella meneghiniana & $\mathrm{F}$ & - & - \\
\hline & Cocconeis sp. & $\mathrm{F}$ & S & S \\
\hline & Tabellaria sp. & $\mathrm{F}$ & - & - \\
\hline \multirow{3}{*}{ Cyanophyta } & Gloecapsa sp. & - & $\mathrm{F}$ & - \\
\hline & Nostoc sp. & $\mathrm{F}$ & - & - \\
\hline & Microcystis aeruginosa & - & $\mathrm{F}$ & - \\
\hline Euglenophyta & Phacus longicauda & - & S & - \\
\hline Crysophyta & Mallomonas sp. & - & S & $\mathrm{F}$ \\
\hline Total species & 33 & 20 & 23 & 17 \\
\hline
\end{tabular}


In-tank 2 (20 fish) (Table 6), a total number of 40 algal taxa were identified in three weather conditions which were distributed over 25 species in wet weather, 23 species in mixed weather, and 22 species in dry weather. The most species was abundance were Ankyra ankora, Coelastrum microporum, Chlorella vulgaris, Haematococcus pluvialis, Lagerheimia ciliate, Pandorina morum, Scenedesmus obliqus, Scenedesmus obtusus, Scenedesmus quadricauda, Staurodesmus dejetus, Asterionella formosa.

Table 6: List of microalgae species in tank two (20 fish) in three weather conditions (Abundance $\mathrm{R}=$ rare occurrence, $<1000$ cells $/ \mathrm{ml}: \mathrm{F}=$ frequent occurrence, $1000-9999$ cells $/ \mathrm{ml} \mathrm{S}=$ subdominant, 10000-99999 cells/ml: $\mathrm{D}=$ dominant, $>100000$ cells $/ \mathrm{ml}$ )

\begin{tabular}{|c|c|c|c|c|}
\hline \multirow{2}{*}{ Division } & \multirow{2}{*}{ Species } & \multicolumn{3}{|c|}{ Weather conditions } \\
\hline & & Wet & Mix & Dry \\
\hline \multirow{33}{*}{ Chlorophyta } & Actinastrum hantzschii & $\mathrm{S}$ & - & - \\
\hline & Ankyra ankora & S & $\mathrm{F}$ & $\mathrm{S}$ \\
\hline & Ankistrodesmus fusiformis & - & - & $\mathrm{S}$ \\
\hline & Botryococcus & - & - & $\mathrm{S}$ \\
\hline & Carteria sp. & $\mathrm{F}$ & - & - \\
\hline & Coelastrum microporum & $\mathrm{F}$ & S & $\mathrm{S}$ \\
\hline & Chlorella vulgaris & S & S & $\mathrm{F}$ \\
\hline & Chlorogonium elongatum & S & - & - \\
\hline & Clamydomonas sp. & & $\mathrm{F}$ & - \\
\hline & Crucigenia quadrata & $\mathrm{F}$ & - & - \\
\hline & $\begin{array}{l}\text { Dictyosphaerium } \\
\text { pulchellum }\end{array}$ & - & $S$ & $S$ \\
\hline & Eustrum biverrucosum & $\mathrm{F}$ & - & - \\
\hline & Golenkinia radiate & S & - & - \\
\hline & Haematococcus pluvialis & $\mathrm{F}$ & $\mathrm{F}$ & $\mathrm{S}$ \\
\hline & Lagerheimia ciliate & S & $\mathrm{F}$ & $\mathrm{F}$ \\
\hline & Monoraphidium consortium & S & S & - \\
\hline & Odeogonium sp. & . & S & - \\
\hline & Oocystis borgei & - & S & D \\
\hline & Pandorina morum & D & D & D \\
\hline & Pediastrum duplex & - & $\mathrm{F}$ & - \\
\hline & Pediastrum selenaea & - & - & $\mathrm{F}$ \\
\hline & Scenedesmus acutus & - & - & $\mathrm{F}$ \\
\hline & Scenedesmus acumitus & - & $\mathrm{F}$ & - \\
\hline & Scenedesmus opaliensis & S & - & - \\
\hline & Scenedesmus obliqus & S & $\mathrm{F}$ & $\mathrm{S}$ \\
\hline & Scenedesmus obtusus & $\mathrm{F}$ & $\mathrm{S}$ & $\mathrm{S}$ \\
\hline & Scenedesmus ornatus & - & - & $\mathrm{F}$ \\
\hline & Scenedesmus dimorphus & S & - & - \\
\hline & Scenedesmus hystrix & $\mathrm{F}$ & - & - \\
\hline & Scenedesmus quadricauda & S & S & $\mathrm{S}$ \\
\hline & Staurodesmus dejetus & $\mathrm{F}$ & S & $\mathrm{F}$ \\
\hline & Tetraedron minimum & $\mathrm{F}$ & $\mathrm{F}$ & - \\
\hline & Ulthrix sp. & - & - & $\mathrm{D}$ \\
\hline Dinophyta & $\begin{array}{l}\text { Sphaerodinium } \\
\text { Woloszynska }\end{array}$ & S & - & - \\
\hline Euglenophyta & Phacus longicauda & - & $\mathrm{S}$ & \\
\hline Crysophyta & Mallomonas sp. & - & $\mathrm{S}$ & $\mathrm{S}$ \\
\hline Cyanophyta & Microcystis aeruginosa & $\mathrm{S}$ & $S$ & $\mathrm{~F}$ \\
\hline \multirow{3}{*}{ Bacillariophyta } & Asterionella formosa & $\mathrm{S}$ & $\mathrm{S}$ & $\mathrm{S}$ \\
\hline & Fragilaria sp. & S & - & - \\
\hline & Cocconeis sp. & - & $\mathrm{F}$ & $\mathrm{F}$ \\
\hline Total species & 40 & 25 & 23 & 22 \\
\hline
\end{tabular}


In-tank 3 (40 fish) (Table 7), a total number of 39 algal taxa were identified in three weather conditions which were distributed over 25 species in wet weather, 24 species in mixed weather, and 15 species in dry weather. Most of the abundance was Chlorella vulgaris, Coelastrum microporum, Golenkinia radiate, Oocystis borgei, Pandorina morum, Asterionella formosa.

Table 7: List of microalgae species in tank three ( 40 fish) in three weather conditions (Abundance $R=$ rare occurrence, $<1000$ cells $/ \mathrm{ml}: \mathrm{F}=$ frequent occurrence, $1000-9999 \mathrm{cells} / \mathrm{ml} \mathrm{S}=$ subdominant, 10000-99999 cells/ml: $\mathrm{D}=$ dominant, >100000 cells $/ \mathrm{ml}$ )

\begin{tabular}{|c|c|c|c|c|}
\hline \multirow{2}{*}{ Division } & \multirow{2}{*}{ Species } & \multicolumn{3}{|c|}{ Weather conditions } \\
\hline & & Wet & Mix & Dry \\
\hline \multirow{30}{*}{ Chlorophyta } & Actinastrum hantzschii & $\mathrm{S}$ & - & - \\
\hline & Ankyra ankora & - & $\mathrm{F}$ & - \\
\hline & Chlorella vulgaris & S & $\mathrm{S}$ & S \\
\hline & Coelastrum microporum & S & $\mathrm{S}$ & D \\
\hline & Clamydomonas sp. & & $\mathrm{F}$ & $\mathrm{F}$ \\
\hline & Chlorogonium elongatum & S & - & - \\
\hline & Crucigenia quadrata & - & $\mathrm{F}$ & - \\
\hline & Dictyosphaerium & - & S & $S$ \\
\hline & Eustrum biverrucosum & $\mathrm{F}$ & - & - \\
\hline & Golenkinia radiate & $\mathrm{S}$ & S & S \\
\hline & Haematococcus pluvialis & - & - & S \\
\hline & Lagerheimia ciliate & S & - & - \\
\hline & Monoraphidium consortium & $\mathrm{S}$ & S & - \\
\hline & Odeogonium sp. & $\mathrm{F}$ & $\mathrm{F}$ & - \\
\hline & Oocystis borgei & S & $\mathrm{s}$ & S \\
\hline & Pandorina morum & S & $\mathrm{D}$ & $\mathrm{D}$ \\
\hline & Pediastrum duplex & S & $\mathrm{F}$ & - \\
\hline & Scenedesmus opaliensis & $\mathrm{F}$ & - & - \\
\hline & Scenedesmus obliqus & $\mathrm{F}$ & - & - \\
\hline & Scenedesmus obtusus & - & $\mathrm{F}$ & S \\
\hline & Scenedesmus ornatus & - & - & $\mathrm{F}$ \\
\hline & Scenedesmus dimorphus & S & - & - \\
\hline & Scenedesmus hystrix & $\mathrm{F}$ & - & - \\
\hline & Scenedesmus quadricauda & S & S & S \\
\hline & Staurodesmus dejetus & $\mathrm{F}$ & - & S \\
\hline & $\begin{array}{l}\text { Staurodesmus } \\
\text { leptacanthum }\end{array}$ & S & $\mathrm{F}$ & - \\
\hline & Staurastrum cyrtocerum & $\mathrm{F}$ & - & - \\
\hline & Staurastrum cingulum & - & $\mathrm{F}$ & - \\
\hline & Stigeoclonium sp. & - & $\mathrm{F}$ & \\
\hline & Tetraedron minimum & - & $\mathrm{F}$ & - \\
\hline \multirow{2}{*}{ Dinophyta } & Sphaerodinium & $\mathrm{F}$ & - & - \\
\hline & $\begin{array}{l}\text { woloszynska } \\
\text { Ceratium sp. }\end{array}$ & - & $\mathrm{F}$ & - \\
\hline Crysophyta & Mallomonas sp. & - & $\mathrm{F}$ & $\mathrm{S}$ \\
\hline \multirow{2}{*}{ Cyanophyta } & Microcystis aeruginosa & - & $\mathrm{F}$ & $\mathrm{F}$ \\
\hline & Chroccocus sp. & $\mathrm{F}$ & - & - \\
\hline Euglenophyta & Phacus sp. & $\mathrm{F}$ & $\mathrm{F}$ & - \\
\hline \multirow{3}{*}{ Bacillariophyta } & Asterionella Formosa & $\mathrm{S}$ & $\mathrm{S}$ & $\mathrm{S}$ \\
\hline & Fragilaria sp. & $\mathrm{S}$ & - & - \\
\hline & Cocconeis sp. & - & S & - \\
\hline Total Species & 39 & 25 & 24 & 15 \\
\hline
\end{tabular}


In-tank 4 (80 fish) (Table 8), a total number of 33 algal taxa were identified in three weather conditions which were distributed over 24 species in wet weather, 21 species in mixed weather, and 20 species in dry weather. The most abundant in three weather conditions were Chlorella vulgaris,
Coelastrum microporum, Dictyosphaerium pulchellum, Haematococcus pluvialis, Lagerheimia cilite, Oocystis borgei, Pandorina morum, Pediastrum duplex, Scenedesmus obtusus, Scenedesmus ornatus, Scenedesmus quadricauda, Staurodesmus dejetus.

Table 8: List of microalgae species in tank four (80 fish) in three weather conditions (Abundance $\mathrm{R}=$ rare occurrence, $<1000$ cells/ml: $\mathrm{F}=$ frequent occurrence, $1000-9999$ cells/ml S = subdominant, 10000 99999 cells/ml: $\mathrm{D}=$ dominant, $>100000$ cells $/ \mathrm{ml}$ )

\begin{tabular}{|c|c|c|c|c|}
\hline \multirow{2}{*}{ Division } & \multirow{2}{*}{ Species } & \multicolumn{3}{|c|}{ Weather conditions } \\
\hline & & Wet & Mix & Dry \\
\hline \multirow{26}{*}{ Chlorophyta } & Ankistrodesmus fusiformis & & - & $\mathrm{F}$ \\
\hline & Ankyra ankora & $\mathrm{F}$ & - & $\mathrm{F}$ \\
\hline & Chlorella vulgaris & $\mathrm{F}$ & $\mathrm{S}$ & S \\
\hline & Coelastrum microporum & S & $\mathrm{S}$ & S \\
\hline & $\begin{array}{l}\text { Dictyosphaerium } \\
\text { pulchellum }\end{array}$ & $\mathrm{F}$ & $S$ & $S$ \\
\hline & Eustrum sp. & $\mathrm{F}$ & - & - \\
\hline & Golenkinia radiate & S & S & - \\
\hline & Haematococcus pluvialis & S & $\mathrm{F}$ & S \\
\hline & Lagerheimia cilite & S & $\mathrm{F}$ & S \\
\hline & Micractinium pusillum & S & - & - \\
\hline & Monoraphidium consortium & $S$ & $\mathrm{~F}$ & - \\
\hline & Odeogonium sp. & & S & - \\
\hline & Oocystis borgei & S & S & $\mathrm{F}$ \\
\hline & Pandorina morum & D & D & D \\
\hline & Pediastrum duplex & $\mathrm{F}$ & $\mathrm{F}$ & S \\
\hline & Scenedesmus acuminatus & $\mathrm{F}$ & - & - \\
\hline & Scenedesmus obliqus & $\mathrm{F}$ & $\mathrm{F}$ & - \\
\hline & Scenedesmus obtusus & $\mathrm{F}$ & $\mathrm{S}$ & S \\
\hline & Scenedesmus ornatus & $\mathrm{s}$ & $\mathrm{F}$ & $\mathrm{F}$ \\
\hline & Scenedesmus quadricauda & $\mathrm{s}$ & $\mathrm{S}$ & S \\
\hline & Staurodesmus dejetus & S & $\mathrm{S}$ & S \\
\hline & Stigeoclonium sp. & & $\mathrm{F}$ & $\mathrm{F}$ \\
\hline & Staurastrum cyrtocerum & $\mathrm{F}$ & - & - \\
\hline & Tetraedron minimum & $\mathrm{F}$ & - & - \\
\hline & Tetraedron muticum & $\mathrm{F}$ & - & - \\
\hline & Ulthrix sp. & - & - & D \\
\hline Bacillariophyta & Asterionella formosa & - & - & $\mathrm{F}$ \\
\hline Crysophyta & Mallomonas sp. & - & $\mathrm{S}$ & $\mathrm{F}$ \\
\hline \multirow{3}{*}{ Cyanophyta } & Microcystis aeruginosa & $\mathrm{S}$ & $\mathrm{S}$ & - \\
\hline & Snowella sp. & - & $\mathrm{S}$ & - \\
\hline & Anabena sp. & - & - & S \\
\hline \multirow{2}{*}{ Euglenophyta } & Phacus longicauda & $\mathrm{F}$ & - & $\mathrm{S}$ \\
\hline & Trachelomonas sp. & - & $\mathrm{F}$ & - \\
\hline Total species & 33 & 24 & 21 & 20 \\
\hline & & $\mathrm{S}=12$ & $S=9$ & $\mathrm{~S}=12$ \\
\hline \multirow{3}{*}{ dominance } & & $D=1$ & $\mathrm{D}=2$ & $\mathrm{D}=1$ \\
\hline & & $\mathrm{F}=6$ & $F=10$ & $F=5$ \\
\hline & & $\mathrm{R}=1$ & $R=0$ & $R=0$ \\
\hline
\end{tabular}


Table 9, showed that the total number of species ranged between 6 to 25 in wet weather, 9 to 24 in mixed weather, and 8 to 22 in dry weather, the number of species was equal in wet and mixed weather which was higher than many species in dry weather, while the total density recorded the highest rate in dry weather (73.21 to 146.50$)$ followed by mix (61.13 to 101.36 ) and wet (11.71 to 46.73$)$. For wet weather, the high density was observed in tank 4 , in mix weather tank 2 , and in dry weather also tanks 2 .

Table 9 Total of Density and numbers of phytoplankton in three weather conditions

\begin{tabular}{ccccccc}
\hline \multirow{2}{*}{ Tanks } & $\begin{array}{c}\text { Wet Weather } \\
\text { Total } \\
\text { species }\end{array}$ & $\begin{array}{c}\text { Total } \\
\text { Density }\end{array}$ & $\begin{array}{c}\text { Mix Weather } \\
\text { Total } \\
\text { species }\end{array}$ & $\begin{array}{c}\text { Total } \\
\text { Density }\end{array}$ & $\begin{array}{c}\text { Dry Weather } \\
\text { Total } \\
\text { species }\end{array}$ & $\begin{array}{c}\text { Total } \\
\text { Density }\end{array}$ \\
\hline control & 6 & 11.71 & 9 & 61.13 & 8 & 73.21 \\
(10 Fish) & 20 & 38.29 & 23 & 70.95 & 17 & 82.62 \\
(20 Fish) & 25 & 30.48 & 23 & 101.36 & 22 & 146.50 \\
(40 Fish) & 25 & 38.66 & 24 & 82.77 & 15 & 126.46 \\
(80 Fish) & 24 & 46.73 & 21 & 77.74 & 20 & 138.94 \\
\hline
\end{tabular}

Figures 1, 2, 3 presents the results of ShannonWeiner, species richness, evenness, and Simpson in control (without fish), T1 (10 fish), T2 (20 fish), T3 (40 fish), T4 (80 fish) for 3 cycles in every weather condition. For Shannon, there was a significant difference between tanks, there were ranged between 0.75 to 1.71 in wet weather, 0.42 to 1.18 in mixed weather, and 0.20 to 1.09 in dry weather. There was no significant difference in species richness between the four tanks but there is a significant difference between them and control there were ranged from 0.89 to 2.07 in wet weather, 1.07 to 2.18 in mixed weather, and 0.82 to 2.08 in dry weather. There is a significant difference in evenness between tanks as they ranged between 0.70 to 0.84 in wet weather, 0.42 to 0.59 in mixed weather, and 0.25 to 0.58 in dry weather. Finally, the Simpson recorded a significant difference between tanks which ranged between 0.47 to 0.71 in wet weather, and from 0.23 to 0.54 in mixed weather, and 0.11 to 0.51 in dry weather.

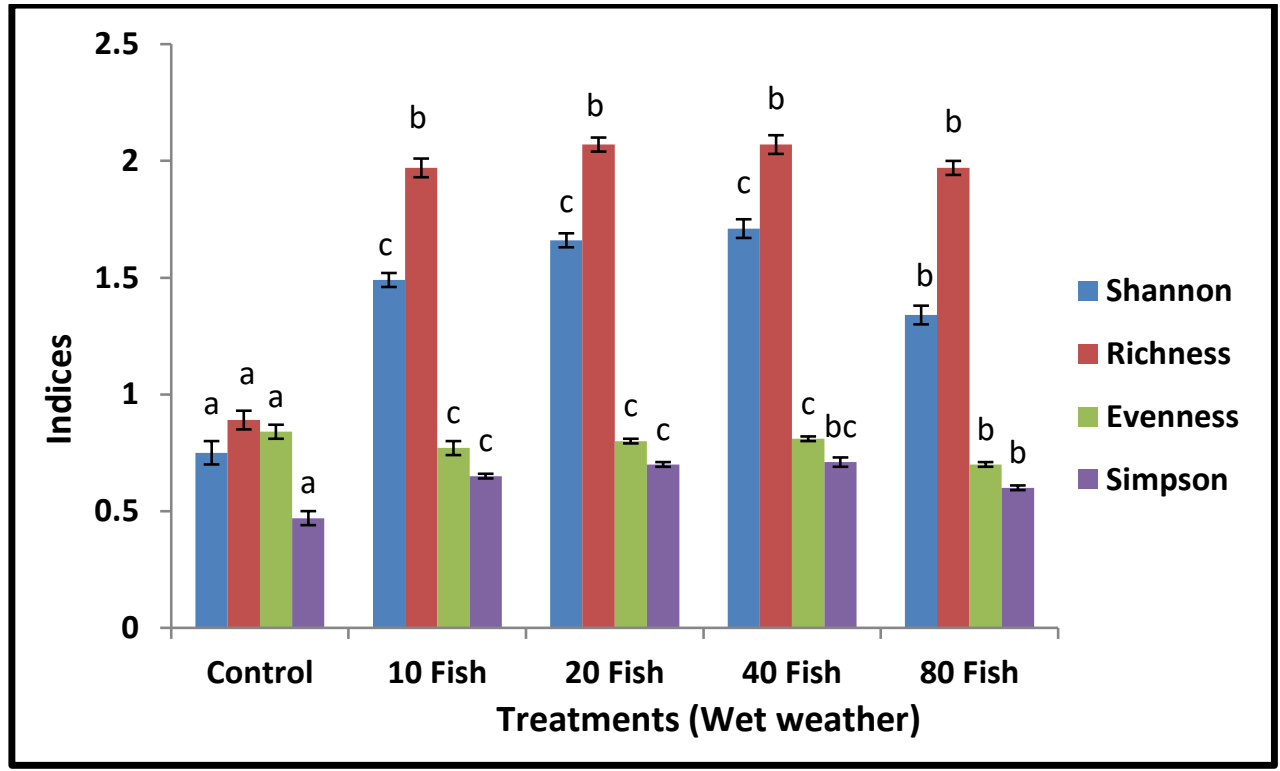

Figure 1: The average of three cycles of Shannon-Weiner, species richness, evenness, and Simpson in different densities of fish from the average of three cycles. Control, T1 (10 Fish), T2 (20 Fish), T3 (40 Fish), T4 (80 Fish) in wet weather conditions. Means with different letters (ac) differ significantly $(p<0.05)$. 


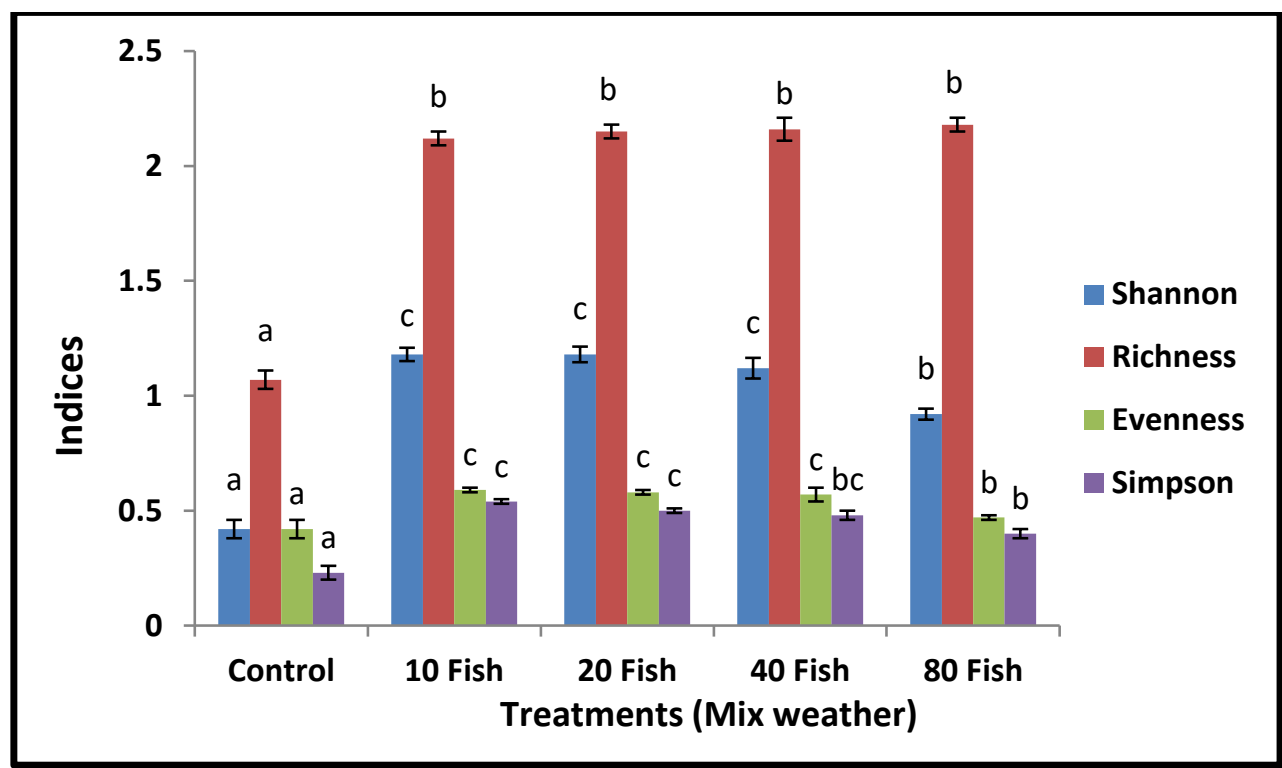

Figure 2: The average of three cycles of Shannon-Weiner, species richness, evenness and Simpson in different density of fish from the average of three cycles Control, T1 (10 Fish), T2 (20 Fish), T3 (40 Fish), T4 (80 Fish) in mix weather condition. Means with different letters (a-c) differ significantly $(p<0.05)$

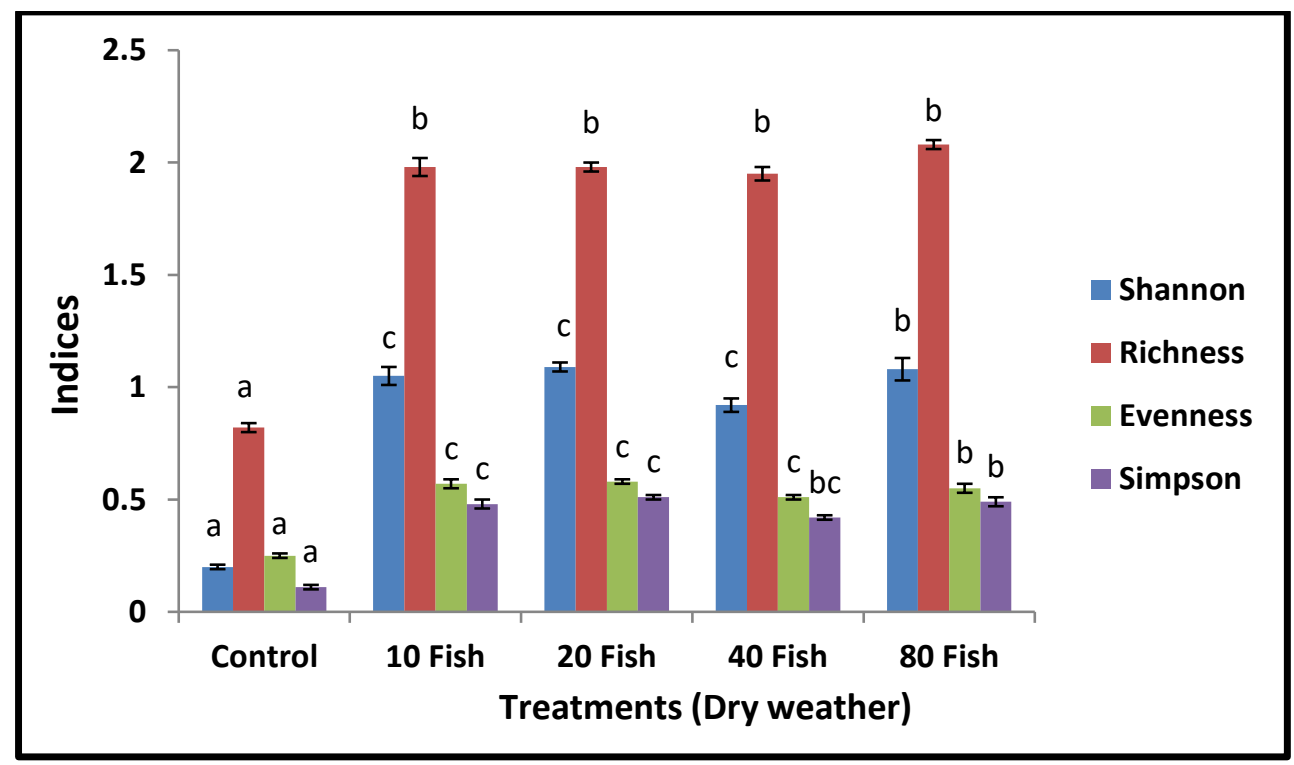

Figure 3: The average of three cycles of Shannon-Weiner, species richness, evenness, and Simpson in different densities of fish from the average of three cycles. Control, T1 (10 Fish), T2 (20 Fish), T3 (40 Fish), T4 (80 Fish) in dry weather conditions. Means with different letters (a-c) differ significantly $(p<0.05)$. 


\section{Discussion}

The findings of this study suggest that there are two possible factors that singly or in a combination that affects species composition and diversity. They are weather conditions and fish stocking density.

To suggest weather conditions have some influence on microalgal composition and diversity is not a straightforward matter because Malaysian weather has no definite pattern. Since there can be cloud cover affecting the light intensity and photoperiod, rain or clear sky and sunshine at any time of the day, week, or month.

Therefore to quantify weather conditions, total daily rainfall, light intensity, and temperature were recorded and ranked. The data indicated that there was a significantly high average rainfall $(\mathrm{p}<0.05)$ in wet weather, moderate rainfall in mixed weather, and low rainfall from one week to the other. The ten days interval was chosen because normally the phytoplankton cycle was between 7-10 days (Round, 1984) which is closer to the week.

Light intensity also followed a similar trend as rainfall, where the average light intensities were significantly lowest in wet conditions, moderately high in mixed weather, and highest in dry seasons.

In the tropic, the light intensity is more likely influenced by the cloud covers or particulate matter from open burning but during this study, there was no episode of haze or open burning that might affect the light intensity.

The mean temperature was marginally lower in wet weather, moderately high in mixed weather, and highest in dry weather conditions but not significantly different $(p>0.05)$ within the range of 26.7-29.4 ${ }^{\circ} \mathrm{C}$. The data suggest that temperature is not critical in the different weather conditions.

Weather scoring is another simple way to assess weather conditions. By observing weather throughout the day, ranking them 1-10 with low rank 1-4 for wet weather, 5 - 7 for mixed weather, and 7-10 for dry weather can adequately describe the weather conditions. Mean weather score for 10 days which is equivalent to one microalgae culture cycle can reveal whether the weather conditions are wet, mixed, or dry.
Another factor that might affect the species composition and diversity of mixed microalgae is fish stocking density and size of fish. Higher fish stocking density will require more food which in turn produces more waste and subsequently more nutrients for the microalgae (North et al., 2006 and Hassan, 2011).

However, the highest fish stocking density will not necessarily give the highest microalgal production because too much-suspended matter limits light penetration to the water body which is essential for microalgae (Parkos et al., 2003).

The results of this study showed that tank without fish did not differ much in term of temperature, $\mathrm{pH}$, TDS, dissolved oxygen, salinity but the Secchi disk depth were significantly higher than tanks with fish.

However, among the tanks with fish, the difference between low stocking density and high fish stocking density is marginal. The differences in water quality as the results of different fish stocking density is so subtle to suggest that they contribute to different microalgae species composition and diversity

Furthermore, there are not many studies regarding the effect of weather conditions and fish stocking densities on phytoplankton population and diversities (Omar et al., 2016). Phytoplankton population studies suggested that weather conditions in terms of the amount of rainfall, weather score, and mean light intensities affected the species composition, diversity, and density.

In wet weather conditions, possible dilution of nutrients in tanks with and without fish reduced available nutrients, thus increasing species composition and diversity (Groendahl and Fink, 2017) but decreasing species density (Cardoso et al., 2012). This is because some species cannot tolerate high nutrient levels (Morris et al., 1979). During wet weather, there is a high incidence of cloud cover that reduces the light intensity (Matuszko, 2012) and duration of optimal photoperiod. There is a close relationship between optimal light intensity, photoperiod, and phytoplankton density (Pal et al., 2013).

In mixed weather conditions, the number of rainfalls, mean weather score, and light intensity 
were intermediate but the species composition and diversity were similar to wet weather conditions but microalgal density was intermediate between wet and dry weather conditions. Many past studies also reported similar observations (Khuantrairong and Traichaiyaporn, 2008; Omar et al., 2016).

In dry weather conditions, the amount of rainfall was the lowest more likely to concentrate the available nutrient in the tanks, highest average light intensity, and more optimal photoperiod allowing some species to grow rapidly while some species are affected. The number of species present and the diversity become low but the densities of microalgae are highest. This phenomenon is also commonly reported in other studies (Kadiri, 2000; Omar et al., 2016).

In-tank without fish, there is limited nutrient available resulting very low number of microalgal species present, low species diversity, and low microalgal density. The nutrient is essential for the existence of microalgae in water bodies irrespective of wet, mixed, and dry weather conditions (Pate et al., 2011; Stubbs, 2016).

This finding indicated those tanks with a stocking density of $10,20,40$, and 80 species have a more or less similar number of microalgal species, diversity, and density in particular weather conditions suggesting that weather conditions have a more profound effect on species composition, species diversity and microalgal densities.

Decrease microalgae density in the rainy season could be due to reduce light intensity, decrease photoperiod, dilution of water due to rain, and lower $\mathrm{pH}$ due to acidic rain. Light intensity another important factor it is considered an important factor to the photosynthesis process to make food then algae growth (Yoon et al., 2008). The light penetrance in water in the dry season leads to improve photosynthesis and reflects on variation in algae species. The growth rates of growing microalgae depend on the light penetration into the microalgae environment (Oswald, 1988). The couler of water and amount of sediments substances and soluble elements affect light penetration and absorption via microalgae.
The highest density of phytoplankton was observed in dry weather which could be as the result of high light intensities, longer period of light duration which allows utilization of available nutrients (Silva, 2005; Badsi et al., 2012), the existence of fish in tank has contributed much to the addition of some nutrients in the water and help phytoplankton to grow. A decrease in microalgae density during the rainy season could be due to reduce light intensity, decrease photoperiod, dilution of water due to rain, and lower $\mathrm{pH}$. This finding was also reported by Nweze (2006).

The phytoplankton divisions in five tanks in the three weather conditions include, Chlorophyta constituted the high rate of total taxon due to flexibility in the physiology, and activities of Chlorophyceae can allow environmental changes better than other species (Silva, 2007). Chlorophyta also can live in a wide range of nutrients and physical environments due to increasing temperature in the dry season could allow the nutrients to become available in the upper layer of a water body. The Bacillariophyta was the second dominant division, while other divisions of phytoplankton exist in some seasons and with insignificant proportions for example Dinophyta, Crysophyta, and Euglenophyta. A Similar assemblage of algae Chlorophyta reported by Ikpi et al, (2013) in the Tropical Earthen Fish Ponds. A high abundance of Chlorophyta was found in wet weather with a moderate temperature (Suresh et al., 2011), this abundance might happen as a result of the cloudy weather and dilution due to rain (Paulette et al., 2011).

Chlorophyta is considered the most prevalent type of algae. The factors of water quality encouraged some species of microalgae to be dominant more than any other species because some of them especially Chlorophyta can tolerate some factors compare with other species. Different phytoplankton species are habitat-specific and their distribution reveals the features of water where they survive (Saha et al., 2000).

Among the species that belonging to Chlorophyta recorded, Pandorina morum can be found in all tanks with different densities of fish in different weather conditions. A high abundance of Pandorina presence in tanks was because of its ability to adapt 
to a wide range of water physico-chemical parameters. For that reason, Pandorina is reported to inhabit a variety of freshwater environments around the world and is common in standing bodies of water such as puddles ponds, and lakes (Van Vuuren et al., 2006).

Bacillariophyta, commonly known as diatoms is common in freshwater and marine habitats. Diatoms are sensitive to environmental conditions changes (Reynolds, 1998). Therefore they can be used as powerful ecological tools to investigate past conditions and monitor environmental changes over time (van Vuuren et al., 2006). Dinopyta species present in high number in the mix and wet weather but missing in dry weather probably due to unfavorable water quality such as high nitrogen or phosphorus. Asterionella formosa was subdominant in three weather conditions in tanks 20 and 40 fish but its presence in tank 4 (80 fish) was fair in dry weather conditions probably due to the presence of small amounts of phosphorus. The presence of small amount of phosphorus may permit the growth of this species. Cyclotella meneghiniana was frequent only in wet weather conditions probably because rainwater dilutes the nutrient in the tank thus allowing this species to exist.

Chrysophyta represented by Mallomonas sp. was present in mix and dry weather but was absent in wet weather probably due to low nutrients, low light, and low temperature during wet weather. Mallomonas sp. is often abundant in water containing high nutrients. Euglenophyta species often live in hard or soft water habitats of varied $\mathrm{pH}$ and light levels also they more abundantly in polluted water (Ersanlı \& Gönülol, 2003). In this study, the presence of Euglenophyta species was subdominant in a tank with high stocking densities in the mix and wet weather probably due to high organic matter and moderate temperature. The presence of this division is not high because it prefers higher organic matter in the water. Phacus longicauda was subdominant in mixed weather conditions in tanks 1, 2, 4 but the occurrence was higher in wet weather conditions.

The distribution of Cyanophyta was diverse between frequent and subdominant in different weather conditions and tanks. Many species of
Cyanophyta produce populations that are toxic to humans and animals so the Cyanophyta is considered an indicator for water quality. It has been observed the growth of some species of this group of algae increased with increasing temperature and organic components especially in tanks with a high stocking density of fish. In this study, Microcystis aeruginosa was found in all weather conditions due to water temperature, dissolved inorganic carbon, and dissolved inorganic nitrogen is considered the main factors controlling growth in various habitats. Similar studies found that water temperature is the most important factor determining the dominance of M. aeruginosa (Imai et al., 2008).

Dinophyta were represented with 3 species and only appeared in wet weather in tanks with 10, 20, 40 fish and in control in dry weather conditions. The existence of diatoms was in wet weather due to certain nutrients is present as a result of rain and this group of algae requires certain amounts of nitrate to grow. Various studies have shown a similar relationship between diatoms and other nutrients, such as iron and nitrate (Takeda, 1998; Eppley \& Renger, 1974). Sphaerodinium woloszynska was present in all tanks.

Some species of microalgae favored low in total dissolved solids, electric conductivity, salinity, and alkalinity in wet weather. Also, high light intensity, alkalinity, temperature, Secchi depth during dry weather conditions in tank 4 with a high stocking density of fish encouraged some species of microalgae to grow such as Botryococcus sp., Ankistrodesmus fusiformis, Scenedesmus acutus, Ulothrix aequalis, Haematococcus pluvialis, Anabaena sp., Peridenium labeled, Dictyosphaerium pulchellum.

The diversity of phytoplankton is linked with several environmental parameters such as temperature, $\mathrm{pH}$, dissolved oxygen, salinity, and nutrients (Sridhar et al., 2006). The highest species diversity was observed in all tanks in the mix and wet weather conditions, these results are consistent with the fact that mix conditions following wet provide an ideal condition for algae to grow, while the lowest was in dry weather conditions due to the impact of high water temperature on due to the 
impact of high surface water on some biochemical process of microalgae species, because of dominating of microalgae species and a limited number of species.

Simpson's index is a measure of diversity that takes into account the number of species present. This index was found to be high in wet and mixed weather conditions and low in dry weather conditions. The value of Simpson's index ranges between 0 and 1 . With this index, the result was nearer to zero (0) shows richness in diversity, and result nearer to one (1) show poor in diversity. On the other hand, the values of species diversity were low in dry weather which may be due to the impact of high surface water on some biochemical processes of microalgae species, because of dominating of some species of microalgae.

The total cell density of microalgae species was diverse between five tanks, the lowest density was in control due to lack of nutrients and absence of fish which is considered a good source for algae feeding and growth. Also, total cell density was high in dry weather conditions with low species diversity. Also, high fish stocking densities result in large populations because of the plant nutrients that are released from fish excrement (Noga, 2011).

The physico-chemical parameter of water in tanks was influenced by the different weather conditions and different stocking densities of fish. Changes in weather conditions influenced water temperature and nutrients, resulting in the declining density of some species of microalgae. The present study showed the differences between different weather conditions which are considered as the main factor impacting phytoplankton with a high number of species in the mix and wet weather and high density in the dry weather. Also, the seasonal succession of microalgae mentioned to Chlorophyta dominated species followed by Cyanophyta, Bacillariophyta, Dinophyta, Chrysophyta, and Euglenophyta. Findings from this study referred to that different weather conditions support Chlorophyta growth more than other divisions. Dominated species physiologically and behaviorally adapted to permanent water column mixing, high turbidity in dry weather. Also stocking density of fish was an important factor because of the effects on cell density of microalgae but at the same time was not affect on species of microalgae; species were close in all tanks.

\section{Acknowledgments}

The author is extremely thankful to Dr. Hishamuddin Omar for his help and his comments.

\section{References}

Abdel-Raouf, N., Al-Homaidan, A., \& Ibraheem, I. (2012). Microalgae and wastewater treatment. Saudi journal of biological sciences, 19: 257-275.

Badsi, H., Ali, H. O., Loudiki, M., \& Aamiri, A. (2012). Phytoplankton diversity and community composition along the salinity gradient of the Massa estuary. American Journal of Human Ecology, 1: 58-64.

Bahadar, Ali and Khan, M. Bilal. (2013). Progress in energy from microalgae: A review, Renewable, and Sustainable Energy Reviews, 27: 128-148.

Barnabé, Gilbert (1994) Aquaculture: biology and ecology of cultured species p. 53, Taylor \& Francis. ISBN 978-0-13-482316-4

Bellinger, E. G., \& Sigee, D. C. (2010). Introduction to freshwater algae. Freshwater algae: identification and use as bioindicators, 140.

Cardozo, K. H., Guaratini, T., Barros, M. P., Falcão, V. R., Tonon, A. P., Lopes, N. P. Colepicolo, P. (2007). Metabolites from algae with economical impact. Comparative Biochemistry and Physiology Part C: Toxicology \& Pharmacology, 146: 60-78.

Edler, L., \& Elbrächter, M. (2010). The Utermöhl method for quantitative phytoplankton analysis. Microscopic and molecular methods for quantitative phytoplankton analysis, 110 .

EPA. (2013). Impacts of Climate Change on the Occurrence of Harmful Algal Blooms. The United States Environmental Protection Agency, EPA 820-S-13-001. 
Eppley, R.W. (1972). Temperature and phytoplankton growth in the sea. Fishery Bulletin 70: 1063-1085.

Eppley, R. W., \& Renger, E. H. (1974). Nitrogen assimilation of an oceanic diatom in nitrogen-limited continuous culture. Journal of Phycology, 10: 15-23.

Ersanl1, E., \& Gönülol, A. (2003). Study on the phytoplankton and seasonal variation of Lake Simenit (Terme-Samsun, Turkey). Turkish Journal of Fisheries and Aquatic Sciences, 3: 29-39.

Goldstein, M. (2002). The complete idiot's guide to weather: Alpha publications, New York.

Groendahl, S., \& Fink, P. (2017). Consumer species richness and nutrients interact in determining producer diversity. Scientific reports, 7, 44869 .

Hassan, A. (2011). Zooplankton as natural live food for three different fish species under concrete ponds with mono-and polyculture conditions. Egyptian J. Aquac, 1(1).

Ikpi, G. U., Offem, B. O., \& Okey, I. B. (2013). Plankton distribution and diversity in tropical earthen fish ponds. Environment and Natural Resources Research, 3: 45-51.

Imai, H., Chang, K.-H., Kusaba, M., \& Nakano, S.i. (2008). Temperature-dependent dominance of Microcystis (Cyanophyceae) species: M. aeruginosa and M. wesenbergii. Journal of Plankton Research, 31: 171-178.

Kadiri, M. O. (2000). Limnological studies of two contrasting but closely linked springs in Nigeria, West Africa. Plant Biosystems, 134(2), 123-131.

Khuantrairong, T., \& Traichaiyaporn, S. (2008). Diversity and seasonal succession of the phytoplankton community in Doi Tao lake, Chiang Mai Province, Northern Thailand. Tropical Natural History, 8(2), 143-156.

Kumar, M., Naik, S. K., Murari, A., \& Rani, Y. (2012). Methods of diatom test: a review of literatures. Journal of Forensic Medicine and Toxicology, 29: 47-54.
Makandar, M. B., \& Bhatnagar, A. (2010). Biodiversity of Microalgae and Cyanobacteria from freshwater bodies of Jodhpur, Rajasthan (India). Journal of Algal Biomass Utilization, 1: 54-69.

Margalef, R. (1958). Information theory in biology. General Systems Yearbook, 3, 36-71.

Matuszko, D. (2012). Influence of the extent and genera of cloud cover on solar radiation intensity. International Journal of Climatology, 32(15), 2403-2414.

New, M. B. (1987). Feed and feeding of fish and shrimp: A manual on the preparation and presentation of compound feeds for shrimp and fish in aquaculture: Food and Agriculture Organization of the United Nations.

Noga, E. J. (2011). Fish disease: diagnosis and treatment: John Wiley \& Sons.

North, B., Ellis, T., Turnbull, J., Davis, J., \& Bromage, N. (2006). Stocking density practices of commercial UK rainbow trout farms. Aquaculture, 259(1-4), 260-267.

Nweze, N. O. (2006). Seasonal variations in phytoplankton populations in Ogelube Lake, a small natural West African Lake. Lakes \& Reservoirs: Research \& Management, 11: 63-72.

Omar, Munay Abdulqadir, Mohamed Amar Naqqiuddin, Shamarina Shohaimi, Hishamuddin Omar, and Ahmad Ismail. (2016). Phytoplankton diversity in relation to different water conditions in two urban manmade lakes. Sustainability, Agri, Food and Environmental Research 4:1-21.

Oswald, W.J., 1988. The role of microalgae in liquid waste treatment and reclamation. In: C.A. Lembi and J.R. Waaland (Eds), Algae and human affairs, Cambridge University Press, Cambridge, 255-281.

Pal, S. W., Singh, N. K., \& Azam, K. (2013). Evaluation of relationship between light intensity (Lux) and growth of Chaetoceros muelleri. Oceanography, 1(3), 1-4.

Parkos III, J. J., Santucci, J., Victor J, \& Wahl, D. H. (2003). Effects of adult common carp (Cyprinus carpio) on multiple trophic levels 
in shallow mesocosms. Canadian Journal of Fisheries and Aquatic Sciences, 60(2), 182192.

Pate, R., Klise, G., \& Wu, B. (2011). Resource demand implications for US algae biofuels production scale-up. Applied Energy, 88(10), 3377-3388.

Paulette, Adon Marie, Ouattara Allassane \& Gourene Germain. (2011). Seasonal variation in the diversity and abundance of phytoplankton in a small African tropical reservoir. African Journal of Microbiology Research, 5: 2616-2626.

Priyadarshani, I., \& Rath, B. (2012). Bioactive compounds from microalgae and cyanobacteria: utility and applications. International Journal of Pharmaceutical Sciences and Research, 3: 4123.

Raghunath, H. M. (2006). Hydrology: principles, analysis, and design: New Age International.

Reynolds, C. S. (1998). The state of freshwater ecology. Freshwater Biology, 39: 741-753.

Round, F. E. (1984). The ecology of algae: CUP Archive.

Ruangsomboon, S. (2012). Effect of light, nutrient, cultivation time, and salinity on lipid production of newly isolated strain of the green microalga, Botryococcus braunii KMITL 2. Bioresource Technology, 109, 261-265.

Saha, S. B., Bhattacharyya, S., \& Choudhury, A. (2000). Diversity of phytoplankton of a sewage polluted brackish water tidal ecosystem. Journal of environmental biology, 21:9-14.

Shaari, A. L., Surif, M., Latiff, F. A., Omar, W. M. W., \& Ahmad, M. N. (2011). Monitoring of water quality and microalgae species composition of Penaeus monodon ponds in Pulau Pinang, Malaysia. Tropical life sciences research, 22: 51.

Shahzad, Umair and Riphah. (2015). Global Warming: Causes, Effects, and Solutions. Durreesamin Journal, 1.
Shannon, C. E., \& Weaver, W. (1949). The Mathematical Theory of Information (Urbana, IL: University of Illinois Press.

Silva, E. (2005). Phytoplankton characteristics, trophic evolution and nutrient dynamics in an urban eutrophic lake: Kandy Lake in Sri Lanka. Restoration and Management of Tropical Eutrophic Lakes (MV Reddy, ed.), 227-270.

Silva, E. (2007). Ecology of phytoplankton in tropical waters: introduction to the topic and ecosystem changes from Sri Lanka. Asian Journal of Water, Environment, and Pollution, 4: 25-35.

Simpson, E. H. (1949). Measurement of diversity. Nature, 163(4148), 688.

Sivasubramanian, V. (2016). Environmental sustainability using green technologies, Hand Book. CRC pres.

Sridhar, R., Thangaradjou, T., Kumar, S. S., \& Kannan, L. (2006). Water quality and phytoplankton characteristics in the Palk Bay, southeast coast of India. Journal of Environmental Biology, 27: 561-566.

Stubbs, M. (2016). Big Data in US Agriculture. Washington DC: Congressional Research Service, Report, 44331.

Suresh, S., Thirumala, S., \& Ravind, H. B. (2011). Zooplankton diversity and its relationship with physico-chemical parameters in Kundavada Lake, of Davangere District, Karnataka, India. Pro-Environment/Pro Mediu, 4: 56-59.

Takeda, S. (1998). Influence of iron availability on nutrient consumption ratio of diatoms in oceanic waters. Nature, 393: 774-777.

Yoon, J.H., Shin, J.H., Ahn, E.K., Park, T.H. (2008). High cell density culture of Anabaena variables with controlled light intensity and nutrient supply. Journal Microbiology Biotechnology, 18: 918-925.

van Vuuren, D. P., Sala, O. E., \& Pereira, H. M. (2006). The future of vascular plant diversity under four global scenarios. Ecology and Society, 11:25. 